\title{
CHALLENGES OF ICT POLICY FOR RURAL COMMUNITIES: A CASE STUDY FROM SOUTH AFRICA
}

\author{
Mpostol Jeremia Mashinini \\ Sithabile Technology Group, South Africa
}

\begin{abstract}
It is alleged that rural people in South Africa are being excluded from the rest of the world in terms of communication and exchange of information due to lack of appropriate ICT policies. This research used grounded theory method to study the local authority of Dr S.J. Moroka in order to understand the limitations of the South African ICT policy for poor rural communities. It found lack of leadership for integrating multiple policy initiatives and evidence of a culture of non-compliance with policies. These are major challenges that need to be addressed. This chapter makes several practical suggestions for the integration of isolated, disadvantaged, and poverty-stricken rural communities into the rest of the world, enabling them to benefit from ICT-related services on a sustainable basis.
\end{abstract}

Keywords: Traditional leadership, grounded theory method, ICT Policies, rural communities, South Africa

\section{Introduction}

Some intellectuals and policy makers question whether poor people living in rural areas in developing countries such as South Africa need to be provided with advanced communication technologies and even warn about the potential harmful effects of attempting to transform poor rural communities into cyber communities [4]. They point out that the provision of clean water, roads, improved primary health care, and schools for teaching people to read and write as a means of improving their quality of life are far more important than providing people in rural communities with computer access to data networks. Nevertheless, the South African Government has been making efforts to provide communication services to rural communities and enable them to be included in an increasingly more electronically connected world.

Hence, this paper seeks to answer the question how can the current ICT policies of the South African Government aiming at the formation of cyber communities in rural areas improve quality of life in these areas? At present South African rural communities are being excluded from the rest of the world in terms of communication, exchange of information, and usage of ICT-related services. 
This chapter seeks to explore existing challenges and provide suggestions for action that may enable the integration of isolated, disadvantaged, and povertystricken rural communities into an increasingly relying on ICT world. The structure of the chapter is as follows. Firstly, the background to the environment where the research was conducted is provided. Secondly, the research methodology used to collect and analyse data is described and justified. Thirdly, there are discussions on key findings such as the current challenges, causes of the challenges, and how these challenges can be corrected. Finally, conclusions drawn from this research are summarised.

It has become obvious that in the face of major challenges such as the total lack of even the most basic infrastructures in a community, namely roads, clinics and schools, that any ICT policy needs to be designed to play a role in addressing or helping to eliminate these critical problems.

\subsection{Background of Dr. S.J. Moroka (research area)}

Dr. S.J. Moroka was identified as a rural area ideally suited to the aims and objectives of this research because of the lack of basic services, lack of suitable infrastructure, and social challenges, among others. It is a rural area that has some exposure to ICT usage and related services on a small scale. It is a local authority situated within Mpumalanga province. The population is estimated between 1 million to 2 million people and it covers an area of about 267,626 hectares. It is governed by a local authority under the leadership of a Mayor (Molefe) and King Mayisha III (Ndebele King). Prior to the 1994 elections, Dr. S.J. Moroka was governed by traditional authorities while administrative activities were carried out by the King and the local Chiefs. During the apartheid regime, few infrastructural development projects were initiated within this area as it was subject to a separate development policy.

This historical situation created a number of challenges that continue to face Dr. S.J. Moroka. These challenges can be summarised as follows: poor infrastructure, unattractive investment opportunities owing to traveling distances, a scattered population, and a shortage of skilled labour. The road network into Dr. S.J. Moroka has suffered from the lack of infrastructural development. Access to even the most basic of services, such as primary health care and education, is difficult due to the lack of transport. Lack of buying power and the extremely slow and low level of economic activity preclude the establishment of a vibrant, service-orientated financial services sector on which to base development. Dr. S.J. Moroka lacks sufficiency in clinics, medical doctors, and other health related services.

This situation forces patients seeking medical help to travel to the nearest clinic and from there they are referred to a distant regional hospital that is servicing communities far beyond its capacity. Cultural and ethnic differences continue to make communication and co-operation difficult in the delivery and administration of key services and projects. A poor economic environment has contributed to the 
high level of abject of poverty within the Dr. S.J. Moroka community. There is a heavy reliance among people on state pensions and children's grants.

According to the Government Report [6], a number of problems exist with regard to the use of ICT in rural areas and attempts to deliver good quality services to all rural people using ICT. Admission of failure to achieve this initiative resulted in the South African Cabinet, led by the President Thabo Mbeki, organising visits to various areas to evaluate whether or not community needs are being met. Prompted by concerns expressed by several rural communities [6], a commission was established within the President's Office headed up by Prof. W. Nkuhla to investigate the problems. The following problems were identified (in no specific order): low level of community literacy, lack of ICT awareness, ICT programs currently implemented do not produce adequate results, inadequate ICT infrastructure, a process of policy formulation that is not suitable for the community needs. Many rural communities share common problems associated with education, health, land, and job opportunities.

\subsection{Landscape of current ICT policies in South Africa}

According to the South African Minister of Communication [8], "the history of ICT policies in SA did not address the needs of all people. Apartheid skewed the manner in which telecommunication infrastructure was established in South Africa". As a result the telecommunication network embodied the characteristics of racial duality. For example, in black formal townships the telephone infrastructure existed, but it was inadequately provided and maintained, while in neighbouring white urban areas the telecommunication infrastructure was well established and supported. In black rural areas, there was a total absence of telecommunication networks [5].

Furthermore, under the previous political dispensation, there was no prospect of rural communities being brought into the mainstream of the conventional telecommunications network as income levels were extremely low and economic activity was often at a standstill. Issues of affordability and privatisation have been priorities for the government to ensure proper empowerment [1].

Convergence within ICT, where current technology allows multiple operations to take place in parallel (such as the ability of a single cable to carry both voice and data) further enhances the potential role to be played by ICT in service delivery to rural communities. In recognising the power of convergence, whereby a single multi-purpose community centre in a rural area can be positioned to serve citizens, the government has recognised the importance of how essential it is to provide accessibility to infrastructure to all people. This has led the government to strive to meet the demand to achieve universal service as urgently as possible. The delivery of universal service is largely dependent on human resource capacity within the industry. Therefore, the National Department of Communications was established to ensure that ICT policies are developed and well implemented. Furthermore, the department was set up to ensure that proper communication and 
dissemination of information from the national government through the Provincial and to the local authorities was carried out. It initiated an internship programme whereby more than 1,000 students are being trained in each ICT sector. The department's mandate includes the provisioning of a "one-stop-service" government information centre for all ICT policies in South Africa to be coordinated centrally [2].

\section{Research methodology}

In this research we used the grounded theory method to address the research question. Grounded theory allows the following benefits. Firstly, it allows a close interaction with the rural community and the researcher. Secondly, the analysis of the different categories by means of identifying properties and dimensions is described together with their relationships and connections. This ensures a better understanding of how to co-ordinate the different relationships. Thirdly, the grounded theory method provides for different types of conditions that address the research question. Fourthly, this method might be more reflective of reality especially the current challenges regarding the status of ICT policies and their leadership that this research seeks to address. Fifthly, the researcher using a grounded theory approach tends to be collaborative and discursive in nature, being in continuous interaction with ideas and in the generation of ideas through constructive criticism and discourse. Sixth, this method has no preconceived ideas; it is shaped and detected by the data from the respondents. Lastly, it is not rigid but flexible as the situation changes. Hence this method set up a framework on how to ensure effective data collection and analysis.

Data was collected through interviews in a number of organisations. The organisations that were selected are those that form part of the rural community and they perform specific roles and provide certain core services. The key organisations that were selected are indicated as follows.

- Organisations that were actively involved in educating and training community members on ICT, community services, and general skills training. These organisations were Siyabuswa Education Improvement Development Empowerment Trust (SEIDET), KwaNdebele Computer Education Centre (KCEC), Kusile Self Help Association for the Disabled (KSHAD), Super Web Trust (SWT), and Nogijima CC.

- Organisations that are involved in church-related services such as preaching the gospel of God, initiating projects through churches, for example, feeding schemes for those who do not have food, donations of clothing and blankets, training people on self-help projects such as gardening, sewing and so on. Such organisations include the Apostolic church, the Methodist and Faith Mission churches.

- Organisations that are involved in political activities such as deciding on key strategic policies and projects will be implemented within the area. Civil organisations form part of the labour movement within political 
groupings that play a role in protecting workers' rights in different working environments. These organisations are the African National Congress (ANC), the Pan African Congress (PAC), the Democratic Alliance (DA), and other smaller local parties such as Sindawonye MaNdebele.

- Traditional structures being all organisations under the leadership of the Kings and Chiefs, governed by traditional authority. They are mainly grouped according to the language spoken, cultural activities practiced, and location. The structures are further responsible for all the traditional and cultural affairs of the community. The leaders were Ngwenyama Mayisha II (the main leader), Chief P Mahlangu, Chief S Mgwezani, and others.

- Economic structures that are mainly involved in business related activities. The main focus being agriculture services where people practice smallscale farming in their own backyards. Small businesses are also assisted in how to grow their businesses to become big corporates through such economic structures. Some of the organisations that are actively involved include the National African Federated Chamber of Commerce (NAFCOC), Small Medium African Farmers Union (SMAFU), and stokvels (an informal way of investing money on a small scale).

- Groups of professionals with different expertise such as nursing, lawyers, medical doctors, police officers, and administrators were part of the selected people. Non-professional individuals, namely, those people who do not have any experience and are called unskilled labour, were also interviewed. The selection of the different organisations and people was considered to be exploratory. Therefore, the research was limited to those organisations and individuals that operated within Dr. S.J. Moroka in terms of services. These comprise the selected groups from which data was collected.

Hence, interviews were conducted according to the different roles of the respondents and ordinary people (users) included in this research. Letters were also sent to all the leaders of the communities explaining the research to be conducted. The letters sent were followed by formal gatherings with all the different leaders, namely, traditional, political, civil society, and the like. The rural community consists of people with diverse cultures and different languages. For example, most of the questions were asked in Ndebele. The researcher had the ability to read and write the same language. As a result, different languages were used when asking questions in order to make sure that all the respondents understood the questions asked.

The analysis followed the open coding technique of grounded theory to uncover, name, and develop concepts. In this way, we opened the text and exposed the thoughts, ideas, and meanings contained therein. Without the first analytical step, the rest of the analysis and communications that follow could not occur. Open coding allowed the data to be broken down into discrete parts, closely examined and compared for similarities and differences. Events, happenings, 
objects, and actions that were found to be conceptually similar in nature or related in meaning were grouped under more abstract "categories".

\section{Discussion of key findings}

The key findings are grouped together in accordance with the main concepts, properties, and dimensions detected in the data. However, the core idea emerging from the grounded theory method is that the leadership required to integrate the different elements is not effective as this stage. For example, the challenges arising from policies not being implemented include a lack of capacity and processes, and the inappropriate recognition of leadership roles. Appropriate leadership is needed to provide the necessary training, ensure implementation, plan strategies, align policies, and consult with all relevant stakeholders. The key findings of our research are described in some detail below.

\subsection{Current status on challenges in rural areas}

The major challenges identified by the respondents were grouped into the following categories. Firstly, the low level of education in rural areas results in a lack of skills to perform key operational activities that could benefit the community. Hence, most of the people living in rural areas are unskilled, while those with skills move to urban areas to seek better work. Furthermore, the high demand for farm labouring means that children under the age of ten often work on farms, and thus receive no education at all. Secondly, the lack of general infrastructure and poor road quality are major challenges. Roads are not designed for heavy vehicle transport. The import and export of goods is thus severely restricted. The existing gravel roads cross streams and are full of potholes, often making it impossible for road users. The electrical infrastructure, installed and supported by ESKOM, is unreliable and unstable; in windy or wet conditions, the power goes off. Furthermore, the present supply does not meet consumer demand, which has resulted in power cuts.

Thirdly, lack of ICT infrastructure within South Africa. In terms of backbone and bandwidth, this infrastructure is provided exclusively by Telkom. However, Telkom has a deployment strategy that concentrates on urban areas as opposed to rural areas. Lack of financial support to maintain the existing infrastructure has become another major obstacle. Thus, the lack of ICT infrastructure negatively impacts on the entire infrastructure and poses a grave threat to the implementation of ICT services in rural areas. Fourthly, lack of environmental scanning to ensure that projects satisfy user requirements and add value to citizens, an appropriate needs analysis is required. It has happened in the past that authorities have established projects which were not needed by the community members and thus there was no return on the money spent.

Fifth, lack of policy implementation. Policies serve as the underlying structure to plan and ensure proper guidance of the different ICT-related services. In the 
current situation not all stakeholders are involved in the development of such policies. Therefore, both the policies themselves and their implementation are inadequate. Most of the projects thus fail in terms of clear deliverables, milestones, associated risks, and so on. Sixth, economic challenges, rural areas are characterised by challenges, principally the lack of financial resources. Funding is limited, if not non-existent, especially for ICT projects. The major challenge for people applying for grants is that they are required to provide guarantees, balance sheets, and previous credit records. Lastly, social challenges. Social challenges directly impact on the daily life of individuals and the community as a whole. The major problem is the high rate of unemployment. Among the consequences of unemployment are poverty, depression, alcoholism, drug addiction, and crime. In situations of poverty diseases arise and spread that further impact on the wellbeing of the community. Although not all poverty-stricken people turn to crime, certain people who have no food to feed their children sometimes resort to theft.

\subsection{Current status on leadership in rural areas}

According to our analysis of the data from the respondents, there are different leadership divisions, such as community leaders, church leaders, traditional leaders, political leaders, etc. Traditional leadership is seen by the community as the principal leadership role, the one which makes things happen. Traditional leaders are currently recognised as the true leaders of the people. In many instances, they are born leaders, not elected by the community, but rather groomed to be good leaders. Being natural leaders, traditional leaders have many followers. One of the reasons why they are so highly respected is that they act in accordance with the will and needs of their people. Traditional leadership is therefore directly linked to the community. This could explain why a project introduced by the local council without endorsement of traditional community leaders might not be adopted. Even though the project may be viable, it first requires the approval of the traditional leadership.

\subsection{Current status on policy development life cycle}

The main driving force that served as the central nervous system of the policies we studied has been the policy development life cycle [2]. The first phase of the life cycle model is the policy development process. This process outlines all the processes that need to be followed when developing any of the policies. The current state is that policy development has reached saturation stage, where there are too many policies being developed almost daily.

The challenges of developing such policies include the capacity to develop policies, the ability to consult with all the relevant stakeholders, and the communication process currently used. At this stage, the policies being developed do not address the needs of the people. They are often seen as irrelevant to the society. 
The second phase is policy implementation. This process is non-existent, particularly in the rural areas. Policies currently are not being implemented due to lack of capacity and the fact that they are irrelevant to the needs of the people.

The third phase is policy monitoring. If policies are implemented, they need to be monitored and measured against defined goals. Monitoring will help to ensure and confirm that they meet their goals. Monitoring will also help to identify gaps during implementation so that adjustments can be made in order to address problems and therefore close gaps. However, our research could not study this stage of the policy process. As policy implementation is non-existent, no monitoring is taking place.

The last phase of the policy process is policy evaluation. Evaluation should be designed to measure and understand the impacts on policy of both internal and external elements such as changes in the political landscape, economic issues, social challenges, internal regulations, and so on. Owing to the fact that policies are not being effectively developed, implemented or monitored, no evaluation is taking place either.

\subsection{Reasons why policies are not implemented}

There are various reasons why policies could not be implemented. This section discusses some of them. The assumption often made about rural people is that they are not educated. Thus they are ignored when it comes to decision making. It is assumed that they cannot add value to the development of policies, even though in the end such policies will affect them. To complicate the challenge, the government, when communicating information via the media, mainly uses Internet services with separate Web sites for the each department. That is where many of the government tenders, vacant positions, departmental services, and so on are announced. Consequently, members of the community are not always aware of what is happening in their own environment. In many instances they just see things develop without being informed or even asked for their input. For example, if the local authority wants to install electricity, they will start digging holes and planting poles without informing the community beforehand. In such instances the community may even resist a project which is beneficial to all. At times a local council would call all people who are living in the same area into a meeting called an Imbizo. The major challenge at such meetings is to prevent the ruling political party from addressing only those issues that directly affect it. The input from opposition parties is not often taken into account. Therefore the Imbizo does not play a meaningful role in the community and is not an appropriate vehicle for distributing information to the community

The South African government is still in the early stages of ICT policy development. There is a shortage of skills in various areas, namely ICT-related services, medical doctors, professional engineers, nurses, and other professions. The major challenge is the lack of skills in policy development, implementation, monitoring, and evaluation. As a result of the skills shortage in the country, policy 
implementation cannot take place. In addition, people have limited knowledge and skills within the policy development framework. Even the government approach does not assist in addressing this challenge. The government has different spheres, namely national, provincial, and local. Each of these spheres have different roles. For example, the local sphere will provide basic community needs and ensure that policies are adopted and used correctly. The provincial sphere will be responsible for implementing and monitoring policies. The national sphere will be mainly responsible for developing, evaluating, and monitoring policies. A problem occurs when officials are not involved throughout the whole process; for example, on a national level when policies are developed that do not include officials from provincial and local government. The government has practically adopted a centralised approach to policy development. In other words, the national government is the sole entity responsible for initiating various policies. The reason why these policies do not work is that most of the policy developers live far away from the people for whom they are developing these very policies. This is a topdown approach, top being the national department in question and down being the local authority and the community.

The Parliament portfolio committees had about thirty-four policies to be finalised during the 2006/7 financial year. In the end, the different committees only managed to finalise ten policies in terms of development. Implementation has not yet started in some and by the time it does there might be a need to amend some of those policies. The main challenge is the lack of an implementation strategy that will guide the process of policy development and ensure its success. Before any policy is finalised, it must be published in the Government Gazette. Only a few people have access to this document and it is not generally available in rural areas. The key challenge is the ability to read and understand how the Gazette works. In certain areas the Gazette is sold, but the local community cannot afford to buy it.

Furthermore, the industry regulators are often the ones that make the situation inoperable. ICASA is one of the regulators that promote competition within the communication industry in network services, VOIP, security, and so on. The giants of the telecommunication industry such as Telkom are there to provide communication services, even to marginalised areas. The motive for issuing communication licenses by the regulator was to introduce effective competition within allocated areas. Even though small companies have been granted permission to operate in a certain areas, the big giants will still compete with them in those areas. In some other instances, there are many regulations such as the Information Technology Act, Chapter 5E Special, that clearly specify the operations of ICT. Such policies were designed by the Department of Public Service and Administration mainly to ensure uniformity within State departments, interoperability, and ability to interface systems and so on. This policy framework - called the SITA house of values - ensures that each State department uses SITA methodology for the procurement and acquisition of other services. However, the framework does not satisfy the needs of all the departments. All the departments 
are still forced to use SITA's services regardless of the value received. The framework was designed five years ago, so much has changed in the meantime. Hence, the framework is irrelevant and not flexible.

\subsection{How can the current situation be improved?}

This research resulted in the following suggestions on how to overcome the above challenges. The assumption often made about rural people is that they are not educated. Indeed, currently about $85 \%$ of the people in rural areas are uneducated and have low-level skills. Therefore, appropriate training programmes need to be designed to address the gaps that have been identified. Furthermore, people need to be educated with respect to human rights and need to be taught how to initiate as well as embark on projects that could help them financially. The education process needs to link up with external challenges. As technology advances, there is a need to match the education process to the current skills set to enable direct and proper usage of technology. Funds need to be invested in the establishment of training centres that will serve as centres of knowledge and as sources of information.

Rural communities, like urban ones, receive vital core services from government departments. To ensure premium quality with respect to service delivery, capacity building and maintenance of sustainable skills are imperatives. There is a shortage of skills in different sectors: ICT, engineering, and science, to name a few. Government needs to train people in the skills that are lacking as part of enhancement of service delivery by the government. Skills transfer initiatives are to be encouraged and promoted; those who have been trained need to ensure that the appropriate skills are taught to fellow staff members.

Rural areas have their own unique challenges. Environmental scanning could help gain insight into the underlying and related dynamics. Amongst others, aspects that need investigation and interrogation when remedial projects are launched incorporate: local challenges, the political situation, economic situation, social, and technology issues. The aspirations of the rural communities, their strengths and weaknesses need to be leveraged to ensure rural community buy-in and successful execution of projects.

Project management principles have to be adopted to ensure the success of any large scale operation. Execution of projects based on best practice often give the assurance that a project will be delivered on time, within budget, and the end product is a fit for purpose, hence ensuring customer satisfaction. The policy development life cycle should not constitute an exception. Policy development initiatives need to adopt project management principles to ensure timely delivery and within given financial and other related constraints. Management of policy development, implementation, monitoring, and evaluation within the context of a project office will give an insightful understanding on related issues and risks and the situation could easily be presented in a dashboard format to enhance interpretation and expedite related decision-making processes. 
To establish a solid ICT infrastructure in rural areas as a prerequisite to the provisioning of ICT-related services requires adequate funding. government needs to create an environment whereby big business will be attracted and encouraged to invest as well as operate in rural areas. Incentives have to be introduced to turn around the current situation where companies are not willing to provide support and maintenance of services in rural areas as the profit margins are either very marginal or non-existent. Government funding could also help local structures such as Non Governmental Organisations (NGOs), Community Based Organisations (CBOs), and civic organisations to identify and promote local talent within the rural areas. There is a dire need for multinational companies like Microsoft, Hewlett Packard, and International Business Machines (IBM), who are directly involved in ICT, to invest in rural communities by providing ICT-related solutions that will benefit the communities. Also, government in its yearly budget allocation needs to make provision for funding in respect of development of ICT in rural areas.

To foster the participation of big business as well as forge the appropriate relationship between big business and government, it might be relevant to establish an ICT forum. The forum could be used to serve as a platform where all key stakeholders responsible for the provisioning of ICT-related solutions could share information, knowledge, and strategies as well. Also, the forum would enable all role players to keep abreast of technological advancements and emerging technologies and initiate development of relevant policies for implementation.

A policy framework is necessary to give a route map to guide the process of policy development life cycle to ensure effective service delivery. Flexibility needs to be built into the framework to accommodate the dynamism of rural environments that are subject to external forces which might be political, economic, and social or labour related in nature. Furthermore, consideration needs to be given to the high rate of unemployment in the country which might imply denial of access to the technology by the intended users as affordability could pose a serious problem.

The policies need to be aligned to the basic needs of the people. Current policies are developed with either minimum or no consultation with affected people; consequently, developed policies do not take into account all the requirements of the people. Testing of policies will ensure that policies are evaluated against the needs of the people. Review of policies needs to be done on a continuous basis to assess their validity and relevance as well as introduce pertinent amendments for implementation.

Implementation of applicable policies and related amendments has to be guided by an implementation strategy. The strategy will outline the strategic objectives to be met and the approach to be adopted in accomplishing the desired end state. The accompanying implementation plan will detail project work packages to be executed, related resource plans, timeframes, and related budgets. 
Emphasis has to be placed on the participation of the affected people to ensure the success of any ICT-related project that has to be implemented. Projects implemented in rural areas are no exception. The people targeted by projects within a rural setting have to be identified from the outset as key beneficiaries as it essential to understand their background, their major challenges, their expectations and know in advance whether a project will address their requirements and be able to manage the expectations of those affected and communicate accordingly.

Successful projects are underpinned by an effective communications strategy. The strategy identifies the content to be communicated, the recipients of various types of communications, the frequency of each communication type as well as the communication channels.

\section{Summary and conclusion}

This chapter focused on the difficulties confronting policy makers attempting to integrate isolated, disadvantaged, and poverty-stricken rural communities into the rest of the world through the provision of ICT-related services on a sustainable basis. It presented findings from a case study of the current ICT policy efforts of the South African Government for the formation of cyber communities in rural areas intended to improve quality of life in these areas.

Our research showed that the policy development process followed in South Africa has reached saturation point, with many policies being developed but never implemented. Hence the main finding of our research is that the implementation of policies is virtually non-existent and consequently there is little policy monitoring and no evaluation.

We found lack of leadership for integrating multiple policy initiatives. Leadership is required to glue all the different elements together and ensure that the environment is scanned in order to understand existing challenges and plan for effective implementation action. Moreover, we found strong evidence of a culture of non-compliance with policies, which is a major challenge that needs to be addressed.

\section{References}

[1] Barendse, A. (2003). Innovative regulatory and policy initiatives at increasing ICT connectivity in South Africa. Netherlands: Elsevier Science Ltd.

[2] Casaburru I, V. (2003). The Input of the Minister of Communications for the parliamentary media briefing, 20 February 2003. Cape Town: Parliament.

[3] Glaser, B. \& Strauss, A. (1967). The discovery of grounded theory: Strategies for qualitative research. New York: Aldine.

[4] Moodley S. (2001) The challenge of e-business for the South African Ltd. Durban: Polity Press.

[5] Morris, M.L. \& Stavron, S.E. (1993). Telecommunication needs and provision to underdeveloped black areas in South Africa, telecommunication policy. Republic of South Africa: Butterworth-Heinmann. 
[6] South African Government (2001). E-government policy. Pretoria: Department of Public Service and Administration,

[7] South African Government (2003). Statistic of South African annual report 2003, Pretoria: Government Printers.

[8] South African Government (2002). Annual report. Cape Town: Government Printers.

[9] Strauss, A. \& Corbin, B. (1998). The basics of qualitative research: Techniques and procedures for developing grounded theory. London: Sage. 